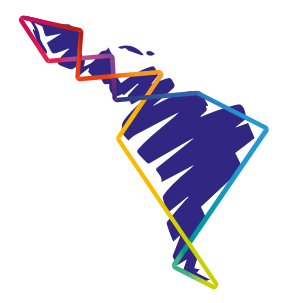

\title{
Revista Latinoamericana de Derechos Humanos: 20 años
}

\section{Revista Latinoamericana de Derechos Humanos: 20 years}

\author{
Evelyn Cerdas Agüero'
}

\section{Resumen}

El objetivo del presente artículo es reseñar 20 años de historia de la Revista Latinoamericana de Derechos Humanos, desde sus inicios como DEHUIDELA en el 2009. Para lograrlo, se realiza un análisis descriptivo con base en la información recabada en los informes presentados anualmente al IDELA, en cada número de la Revista y en la página web de esta. Se propone que esta publicación nace como un esfuerzo del Instituto de Estudios Latinoamericanos (IDELA) para apoyar el programa de Derechos Humanos, así como una oportunidad para desarrollar diversas temáticas que estimularan al estudiantado del Instituto en sus orientaciones de investigación. A partir de un nuevo esfuerzo en el 2009, la Revista da un paso para convertirse en una publicación científica indexada, cuyo eje central es la difusión de estudios vinculados con los derechos humanos desde diversas disciplinas y enfoques. Se concluye que la labor desarrollada a lo largo de los años ha logrado que la Revista Latinoamericana de Derechos Humanos sea una publicación impresa y electrónica de calidad que aporta a la difusión del conocimiento de los derechos humanos en América Latina desde la universidad pública.

Palabras clave: Revista Latinoamericana de Derechos Humanos, publicación científica, IDELA, derechos humanos.

\section{Summary}

This paper aims to review 20 years of history of the Revista Latinoamericana de Derechos Humanos (Latin American Journal of Human Rights) since its inception as DEHUIDELA in 2009. The article proposes that this publication is born as an effort of the Instituto de Estudios

1 Doctora en Educación, Máster en Derechos Humanos y Educación para la Paz, directora-editora de la Revista Latinomericana de Derechos Humanos. Académica del Instituto de Estudios Latinoamericanos (IDELA), Universidad Nacional, Costa Rica. 
Latinoamericanos (Institute of Latin American Studies) (IDELA) to support the Human Rights program, as well as an opportunity to develop various themes that will stimulate the students of the Institute in their research topics. Starting with a new effort in 2009, the journal takes a step to become an indexed scientific publication whose main focus is the publication of works related to human rights as perceived from various disciplines and approaches. The work input over the years has made Revista Latinoamericana de Derechos Humanos a quality print and electronic publication that contributes to the dissemination of knowledge of human rights in Latin America from the public university.

Keywords: Revista Latinoamericana de Derechos Humanos; Scientific publication; IDELA; Human rights.

\section{Introducción}

En este trabajo se pretende reseñar parte de los 20 años de historia de la Revista Latinoamericana de Derechos Humanos desde sus inicios como DEHUIDELA, en el 2009, proyecto que ha formado parte de la visión del Instituto de Estudios Latinoamericanos (IDELA), Facultad de Filosofía y Letras de la Universidad Nacional (UNA), Costa Rica. Para ello se ha realizado un análisis descriptivo con base en la información recabada en los informes presentados anualmente al IDELA, en cada número de la Revista y en la página web de esta.

La Revista Latinoamericana de Derechos Humanos representa una puerta para socializar el conocimiento desde la UNA, es un hito en el compromiso de esta Universidad para aportar al desarrollo del conocimiento. Es un aporte de la Facultad de Filosofía y Letras para participar en la difusión del conocimiento acorde con su misión de crear, difundir y beneficiarse del conocimiento así como promover "los enlaces e interrelaciones entre esos conocimientos, con el fin de que la comunidad humana... alcance etapas superiores de convivencia social y material." (Misión FFL, 2017). además, se enfoca en la misión del Instituto en la promoción del respeto de la dignidad humana, y en la construcción de una sociedad más justa, libre y respetuosa de los derechos humanos.

Asimismo, se enmarca en las áreas de estudio del IDELA que se encuentran ligadas a las situaciones y realidades referentes a los derechos humanos en América Latina, en tanto que este campo representa, para el IDELA, un desafío y a la vez un compromiso en la formación, creación de conciencia, divulgación, vivencia y promoción de los derechos humanos. De esta manera, el Instituto adquiere especial importancia en la promoción de los derechos humanos en un entorno de educación, investigación y extensión, dentro y fuera del ámbito universitario. En este sentido, es importante destacar que la Revista se enmarca 
dentro de la misión del IDELA, dirigida a contribuir "al proceso de construcción de una América Latina, entendida como unidad en la diversidad cultural, abordando lo creativo a partir de una lectura objetiva y una visión propia de nuestra realidad" (IDELA, 2017, parr. 1) y como un espacio potenciador de esfuerzos académicos comprometidos con este proceso, con el propósito de contribuir al "cultivo de una lógica de pensamiento histórico latinoamericano que profundiza en el conocimiento de la realidad de los pueblos latinoamericanos y propulsa transformaciones sociales significativas desde un sentido de aprendizaje crítico." (IDELA, sf, párr. 3

Ante este panorama, la Revista se orienta a ser una herramienta académica, formativa, de conocimiento, informativa, de análisis crítico, de difusión, y un espacio participativo para compartir los aportes del quehacer crítico, analítico, de promoción y de reconocimiento de los derechos humanos en América Latina.

\section{Acerca de los orígenes: DEHUIDELA}

La Revista Latinoamericana de Derechos Humanos surge en 1999 con el nombre de Derechos Humanos IDELA conocida por sus siglas como DEHUIDELA, como parte del proyecto "Módulos Sistematizados en Derechos Humanos" en el IDELA. Marta Morera, académica del Instituto, en esa época, tuvo la visión de iniciar este proyecto como una forma de apoyo a los programas de posgrado del Instituto: la Maestría en Derechos Humanos y Educación para la Paz, la Maestría en Estudios Latinoamericanos y la Licenciatura en Estudios Latinoamericanos, así como a los programas de extensión e investigación. Para quien tuvo esta idea, una publicación en el área de los derechos humanos vendría a ser una oportunidad para desarrollar diversas temáticas que estimularan al estudiantado en sus orientaciones para la investigación.

La publicación de ese momento tuvo como objetivo principal investigar en las fuentes técnicas especializadas temas de derechos humanos para obtener información, la cual, una vez sistematizada, diera paso a la publicación de dos productos, el Módulo (que es la primera versión de la revista DEHUIDELA) y una Antología, de forma que la iniciativa generara publicaciones con fines didácticos que fortalecieran las áreas de docencia, investigación y extensión del Instituto.

La recopilación de la información se realizaba con base en dos criterios: la pertinencia y la periodicidad, con la posibilidad de repetir temas para ampliarlos y actualizarlos. Durante los primeros 9 años se realizó una labor de publicación de las antologías monotemáticas referentes a derechos humanos y DEHUIDELA, los cuales eran cuadernillos monotemáticos también sobre derechos humanos. Estos se distribuían 
en diversos centros de documentación de América Latina y despertaron un interés respecto a las diversas temáticas abordadas.

Se publicaban dos DEHUIDELA y una antología anualmente. Las publicaciones monotemáticas abarcaron diversos temas entre los cuales se pueden mencionar: los derechos de afrodescendientes, migrantes, mujeres, personas con discapacidad; la educación en derechos humanos; el acceso a la educación de las niñas; el trabajo infantil; mujeres y trabajo sexual, entre otros.

Así, se puede decir que DEHUIDELA inició como la primera publicación periódica en una universidad pública en Costa Rica que abordó, de forma particular, el tema de los derechos humanos desde 1999.

Con los cambios presentados en el contexto latinoamericano, las transformaciones vinculadas a las tecnologías de la información y el interés de IDELA por incidir en la realidad latinoamericana a través de la difusión del conocimiento científico, en el 2009 se compromete en un nuevo proceso para que la publicación se transforme en una revista científica en el ámbito de los derechos humanos. Esta renovación inició a cargo de la editora y directora Evelyn Cerdas Agüero, académica del Instituto. Es así como la publicación cambia su nombre a DEHUIDELA: Revista de Derechos Humanos (nombre con el cual salieron 2 números, el 17 y el 18) para posteriormente llamarse Revista Latinoamericana de Derechos Humanos (a partir del número 19 en el 2009).

\section{La primera época: DEHUIDELA}

Los primeros 9 años de la Revista, DEHUIDELA, de 1999 hasta el primer semestre del 2008 salieron 16 números, 2 por año (con excepción del año 2000) en formato impreso a cargo de la editora Marta Morera Salas hasta el número 13, y del 14 al 16 a cargo de la editora Evelyn Cerdas Agüero. Estos números correspondían a una compilación de textos vinculados a temas específicos de derechos humanos con la mención de cada uno de los autores o autoras.

Los primeros números estaban compuestos por 6 apartados, a saber: artículo introductorio, en este se incluía un artículo sobre la fundamentación teórica y filosófica de los derechos humanos; el IDELA y los derechos humanos, con textos sobre el Programa de Derechos Humanos y Educación para la Paz del Instituto; organismos que trabajan sobre derechos humanos, versaba sobre textos que incorporaban el trabajo de organizaciones gubernamentales y no gubernamentales en el ámbito de los derechos humanos; direcciones sobre derechos humanos, en este apartado se 
ofrecía una lista de direcciones electrónicas en las que se podía recabar más información sobre el tema abordado; artículos temáticos sobre derechos humanos, noticias y documentos sobre derechos humanos, estos dos últimos apartados incluían noticias y documentos relativos al tema específico del número.

A partir del número 17 la Revista cambia su nombre a Revista de Derechos Humanos y posteriormente a Revista Latinoamericana de Derechos Humanos, en la cual ya no se incorporan los apartados que se venían publicando, sino solo artículos originales y no textos compilados de la web; además, a partir de este número, ya no se realizan las publicaciones monotemáticas. El siguiente cuadro muestra los temas de los números correspondientes a los primeros 9 años:

Tabla 1

Temas de DEHUIDELA 1999-2008

\begin{tabular}{|c|c|c|c|}
\hline Tema & Número & Año & Fecha \\
\hline Varios temas & 1 & 1 & Enero-abril, 1999 \\
\hline Varios temas & 2 & 1 & Mayo-julio, 1999 \\
\hline $\begin{array}{l}\text { Educación, trabajo, salud y los derechos } \\
\text { humanos }\end{array}$ & 3 & 1 & Agosto-octubre, 1999 \\
\hline Derecho internacional humanitario & 4 & 2 & Agosto-octubre, 2001 \\
\hline Migración y derechos humanos & 5 & 2 & Noviembre-diciembre, 2001 \\
\hline Ambiente y derechos humanos & 6 & 3 & Enero-diciembre, 2002 \\
\hline Derechos de los civiles en guerra & 7 & 4 & Enero-diciembre, 2003 \\
\hline Derechos humanos de los pacientes & 8 & 5 & Enero-abril, 2004 \\
\hline Derechos humanos y democracia & 9 & 5 & Mayo-diciembre, 2004 \\
\hline Afrodescendientes & 10 & 6 & Enero-junio, 2005 \\
\hline Seguridad alimentaria & 11 & 6 & Junio-diciembre, 2005 \\
\hline Derecho a la cultura & 12 & 7 & Enero-mayo, 2006 \\
\hline $\begin{array}{l}\text { Derecho al trabajo de las personas } \\
\text { adultas mayores }\end{array}$ & 13 & 7 & Junio-diciembre, 2006 \\
\hline Derecho a la educación & 14 & 8 & Enero-junio, 2007 \\
\hline Educación en derechos humanos & 15 & 8 & Mayo-agosto, 2007 \\
\hline La educación de las niñas & 16 & 8 & 2008 \\
\hline Revista de derechos humanos & 17 & 9 & Enero-junio, 2008 \\
\hline Revista de derechos humanos & 18 & 9 & Julio-diciembre, 2008 \\
\hline
\end{tabular}

Fuente: elaboración propia. 


\section{Revista Latinoamericana de Derechos Humanos: Una publicación científica}

A partir del 2009, la Revista inicia un período de transformación para formar parte de las revistas científicas de Costa Rica y América Latina, especializada en el tema de los derechos humanos. Así, los objetivos de la Revista se centran en abrir un espacio de reflexión, discusión, análisis crítico y propuestas en el área de los derechos humanos desde diferentes disciplinas con énfasis en América Latina; además, difundir la producción y los aportes en las áreas relacionadas con los derechos humanos que realiza el IDELA, la población académica y estudiantil de la UNA; así como personas y organizaciones que trabajan en el tema de los derechos humanos en Costa Rica y América Latina.

De esta forma, la Revista se ha especializado en la publicación de trabajos cuyo eje central son los derechos humanos desde diversas disciplinas y enfoques, responsabilidad que asume a través del establecimiento de una serie de criterios de calidad y rigurosidad que le permitan posicionarse, de forma seria, como una publicación periódica y científica que aporta desde la universidad pública.

A partir del 2009 el trabajo se ha centrado en fomentar un proceso que permitiera que la publicación estuviera caracterizada por la calidad, para lo cual fue necesario establecer una gestión de calidad respaldada en pautas editoriales vinculadas a la presentación, gestión y a los contenidos de los textos, elementos que permitieran rigurosidad científica. Aunado a una serie de elementos de formato y de normas internacionales entre los cuales se puede mencionar el ISSN para la versión impresa (ISSN: 1659-4304) y para la versión electrónica (EISSN: 2215-4221), la Revista inicia su respaldo con un Consejo Editorial constituido por cinco miembros, un Consejo Internacional compuesto por siete miembros y con el sello editorial de la Editorial de la Universidad Nacional (EUNA).

Con los años, la Revista ha mejorado y ha logrado una mayor visibilidad a través de un trabajo de difusión, por medio de actividades institucionales, distribución de la versión impresa, pertenencia a redes y la puesta en marcha del Portal de Revistas Académicas de la Universidad Nacional a partir del 2014, en el cual la revista tiene su página web (https://www.revistas.una.ac.cr/derechoshumanos). El Portal ha sido una oportunidad para el paso de la publicación impresa a la electrónica, de tal forma que la Revista cumple con los criterios de calidad de una publicación electrónica, es parte del acceso abierto y ofrece los textos completos para su descarga. 
Además de esto, se ha buscado, de forma constante, mejorar e incluir criterios para formar parte de directorios, bases de datos e índices. Así se encuentra en los siguientes índices: DOAJ (Directory of Open Access Journals) y Latindex (Sistema Regional de Información en Línea para Revistas Científicas de América Latina, el Caribe, España y Portugal); en los directorios: CIRC (Clasificación Integrada de Revistas Científicas), OEI - CREDI (Biblioteca Digital de la Organización de Estados Iberoamericanos), IBSS (International Bibliography of the Social Sciences); en las bases de datos: ERIH PLUS, Actualidad Iberoamericana, Journal TOCs (The latest Journal Tables of Contents), Journals for Free (Directorio de revistas de acceso abierto), MIAR (Matriz de Información para la Evaluación de Revistas), OAJI (Open Academic Journal Index), REDIB (Red Iberoamericana de Innovación y Conocimiento Científico, sustituye E-Revist@s), SHERPA/ ROMEO (Políticas de derechos de autor y autoarchivo), CIRC (Clasificación Integrada de Revistas Científicas), Sicultura (Sistema de Información Cultural de Costa Rica); en los motores de búsqueda: Google Académico (Google Scholar), Google Analytics, BASE (Bielefeld Academic Search Engine); portales: Portal electrónico de Revistas Académicas de la Universidad Nacional, Portal de Portales Latindex, WWS (WorldWideScience); repositorios: Repositorio de la Universidad Nacional, Kimuk (Repositorio Nacional de Costa Rica) y el Repositorio La Referencia.

Es importante destacar que el auge de la Revista se debe al trabajo del equipo técnico y editorial, al apoyo del Consejo Editorial, a la colaboración de pares evaluadores y de autores y autoras, a la EUNA; además, al apoyo que ha dado el IDELA para mantener el proyecto vigente por 20 años, así como el aval y acompañamiento de la Dirección de Extensión de la Universidad Nacional, a la cual estuvo adscrito por muchos años, y, en la actualidad, a la Vicerrectoría de Investigación.

\section{Sobre las publicaciones 2009-2019}

Desde el 2009 hasta el primer semestre del 2019 se han publicado 12 volúmenes de la Revista, 22 números de los cuales 1 es un número extraordinario (Tendencias de las migraciones) y otro es número doble. En estos 22 números se incluyen 233 textos en total, de los cuales 220 corresponden a artículos, 9 a poemas y 4 a reseñas. De estos datos también se deriva que 46 de los textos publicados son en coautoría de dos o más autores y autoras.

En lo que se refiere a las autorías, 283 personas han participado como autoras, 169 corresponden a mujeres y 120 a hombres como se denota en la Figura 1, cuyo gráfico incorpora los datos desde el 2009 al 2019. Los números en los cuales hay 
mayor cantidad de mujeres autoras corresponden al Vol. 29 núm. 1, 2018 con un 79 \% y al Vol. 23 Núm. 2, 2012, lo cual equivale a un 78 \% del número con 14 autorías; los siguientes corresponden al Vol. 25 núm. 2, 2014 con un 62 \%; el Vol. 26 Núm. 1. 2015 con 62 \%; el Vol. 30 núm. 1. 2019 con 58 \% y 11 autorías de mujeres en el Vol. 24 núm. 1-2 con un 52 \%, 2013. Los números que tienen más autorías de hombres son el Vol. 22 núm. 2. 2011, con un 61 \%, Vol. 26 núm. 2. 2015, con $50 \%$, Vol. 22 núm. 1.2011 con un $54 \%$.

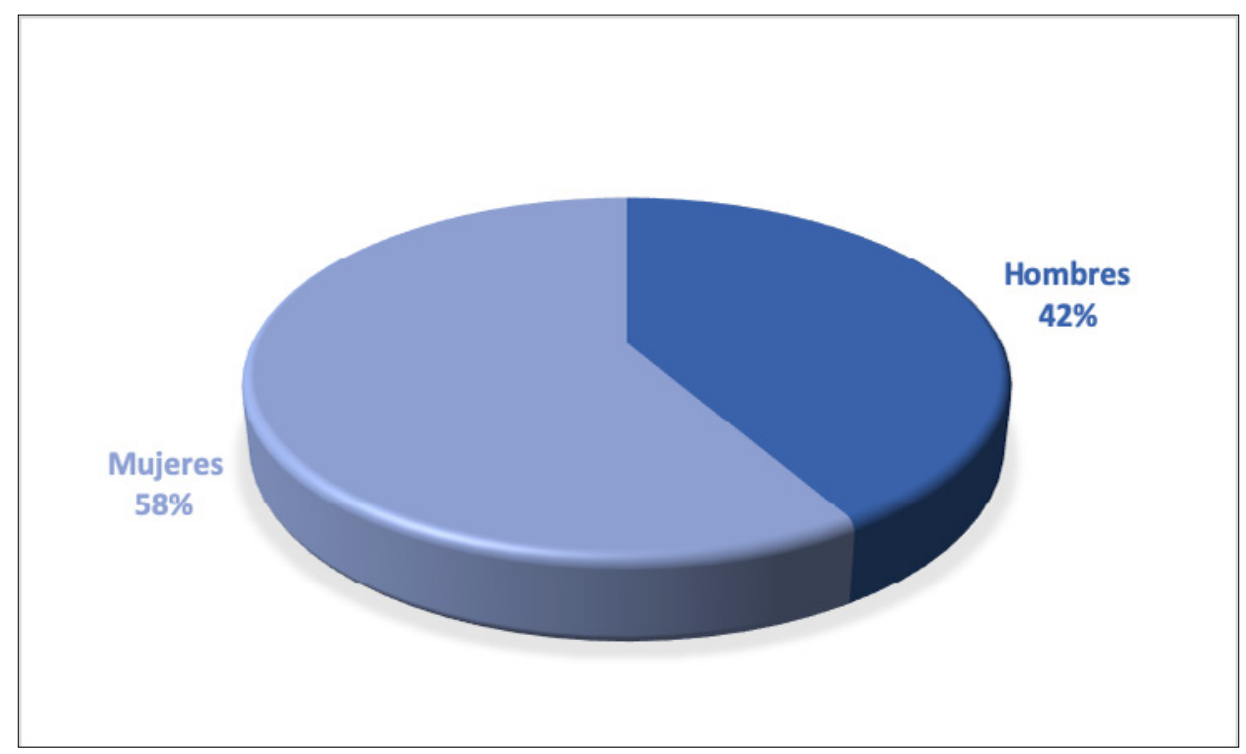

Figura 1. Porcentaje de autorías de la Revista por género. Elaboración propia.

En lo referido a los países de procedencia de las personas que contribuyen en la revista como autoras, estas son de América Latina y España, el 28 \% (80) son costarricenses (del país de edición), el 72 \% son externas al país de edición de la revista, representado por procedencia argentina (42), colombiana (38) y española (37), chilena (22), brasileña (22), mexicana (16), venezolana (11) y de otras nacionalidades (22) de países como Perú, Uruguay, Ecuador, Paraguay, Guatemala, Italia, Nicaragua, Panamá, Alemania, Polonia, Bolivia y Puerto Rico. En la Figura 2 se muestran los porcentajes de la procedencia de autores y autoras del 2009 al 2019. 


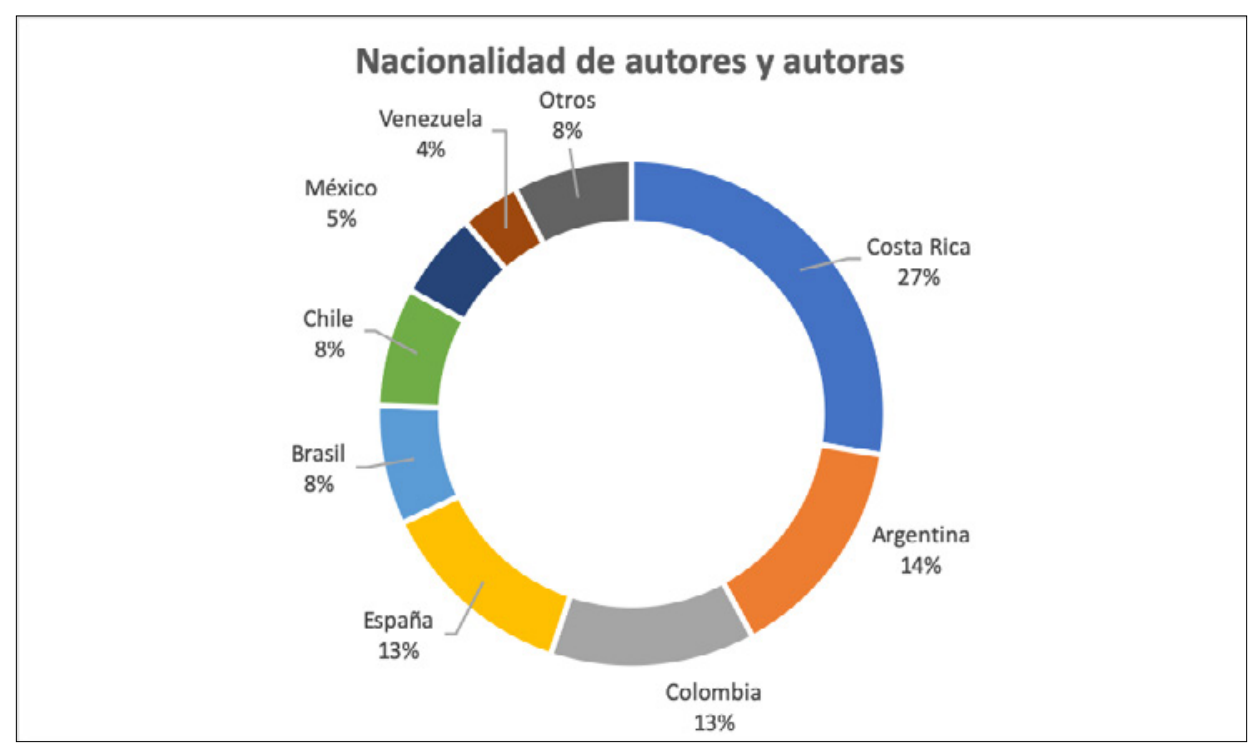

Figura 2. Porcentaje de autorías de la Revista por país de procedencia. Elaboración propia.

En lo referente a las instituciones de adscripción de los autores y autoras, que brindan esa información, aproximadamente 220 pertenecen a universidades, el restante, 69, se distribuyen en adscripciones a ONG, Ministerios de Justicia, grupos de investigación, IIDH, CONICET, FLACSO, CONACYT, comisiones de derechos humanos, instituciones de secundaria, como lo muestra la Figura 3. En lo que corresponde a las disciplinas de procedencia de autores y autoras es importante recalcar que algunos tienen varios títulos, sin embargo, se adscriben a una profesión específica sin dejar de mencionar las otras. A nivel general se puede hablar que las profesiones de los autores y autoras de la revista en su mayoría provienen de áreas del derecho, de la educación (como académicos y académicas de universidades) y de relaciones internacionales; en menor número de áreas como la psicología, ciencias de la comunicación, derechos humanos, ciencias sociales, estudios latinoamericanos y sociología. 


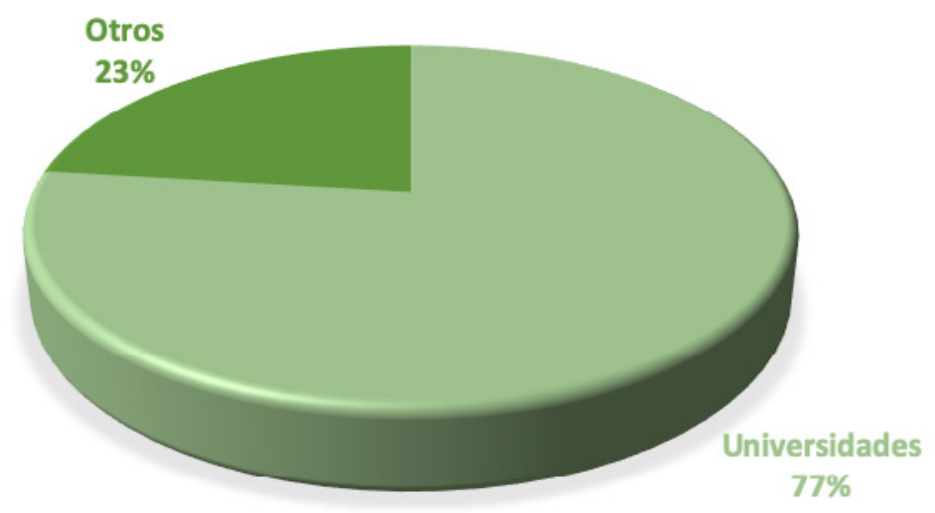

Figura 3. Instituciones de adscripción de autorías de la Revista. Elaboración propia.

\section{Temáticas de la revista}

Los textos que se publican en la Revista tienen como eje central los derechos humanos que abarcan diversas perspectivas vinculadas a este campo. Se incluyen textos con abordajes teóricos o jurídicos, los cuales no se refieren a poblaciones de estudio específicas; también incorpora textos como estudios de caso, experiencias didácticas o de extensión y resultados de investigación. Los temas de los trabajos publicados en su mayoría se refieren a personas migrantes, niñez e indígenas, en menor grado mujeres y juventud, y a otros grupos menos incluidos como personas adultas mayores, afrodescendientes, privadas de libertad, personas con alguna condición de discapacidad y grupos universitarios de estudiantes. Así, resulta importante considerar la necesidad de promover la recepción de textos en los cuales se aborden las poblaciones que participan en menor grado y otras que no se han incluido.

Además, tratan temas referentes a países específicos. Se puede mencionar que la mayoría de textos que incluyen países como temas de estudio hacen referencia a 4 países principales: Argentina, Costa Rica, Colombia y México; seguidos de Venezuela, Chile y Brasil, así como los referentes a la región de Centroamérica y América Latina en general; además, de otros países, en menor número, como Ecuador, Guatemala, Cuba, Uruguay, Puerto Rico, Estados Unidos y España, y dos regiones: Europa y África. Lo anterior se vincula con las nacionalidades de los autores y las autoras, pues, así como la concentración de temas vinculados están en Argentina, Costa Rica y Colombia, lo mismo sucede con la procedencia de las personas autoras. 


\section{Sobre la distribución y estadísticas de visitas de la revista}

La Revista Latinoamericana de Derechos Humanos se distribuye en su formato impreso en 57 centros de documentación en 13 países de América Latina y el Caribe, además de Estados Unidos, Alemania, España, Bélgica, Inglaterra y Francia.

En formato electrónico la Revista es de acceso abierto (licencia Creative Commons BY-NC-ND: Creative Commons Reconocimiento-NoComercial-SinObraDerivada 3.0 Costa Rica License), está disponible en línea en la página institucional de Revistas Académicas de la Universidad Nacional (https://www.revistas.una.ac.cr/); esto ha permitido recabar información de las visitas a través de las "Estadísticas de visitas" de Google Analytics.

Con respecto a las visitas, desde el año 2011 (año en el que inició el proyecto del portal de revistas) hasta el 2019 se contabilizan 199,339 visitas, de las cuales un $22 \%(43,763)$ provienen de Costa Rica y un 77,7\% $(154,804)$ de otros países, en su mayoría de Argentina (31,53\%), México (19,35\%) y Colombia (11,64 \%) (Figura 4).

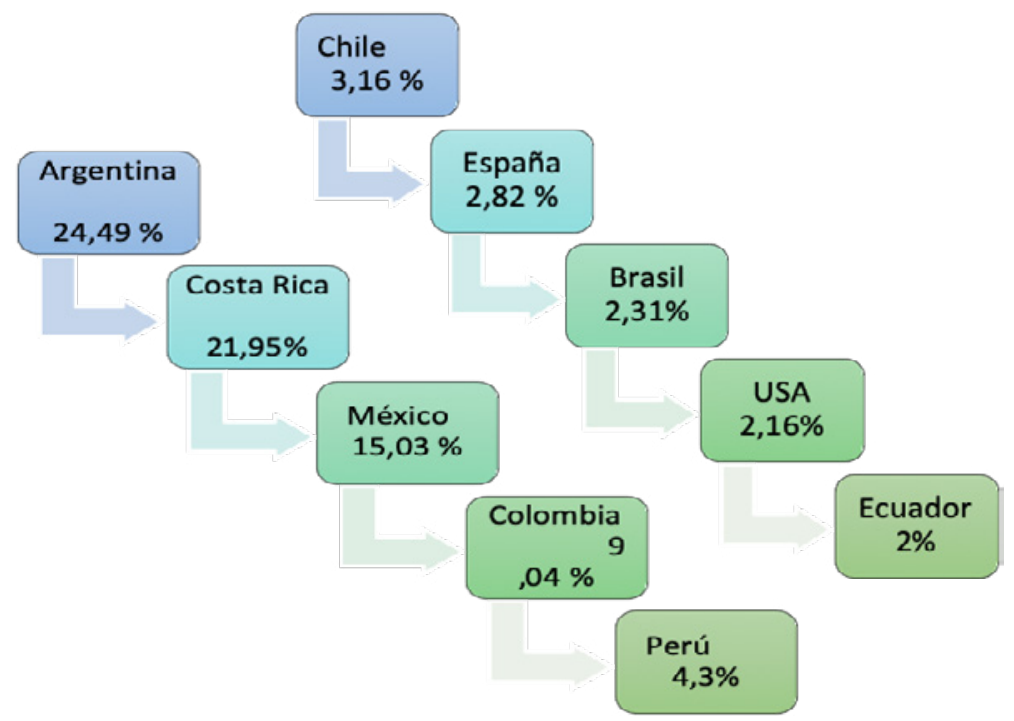

Figura 4. Visitas a la Revista por país. Estadísticas de visitas, según Google Analytics, 2019.

En la siguiente tabla se muestran las visitas anuales a la revista en su versión electrónica desde el julio del 2011 hasta diciembre del 2019, siendo el 2018 el año con mayor cantidad, en este también se puede ver como estas han aumentado 
con el pasar de los años, además, el cuadro muestra los porcentajes de visitas en comparación con Costa Rica.

Tabla 2

Visitas por año versión electrónica de la RLDH

\begin{tabular}{|c|c|c|c|}
\hline Año & Cantidad de visitas & Países & Porcentajes \\
\hline \multirow{2}{*}{2011} & \multirow{2}{*}{2316} & Costa Rica & $42,8 \%$ \\
\hline & & Otros & $57,1 \%$ \\
\hline \multirow{2}{*}{2012} & \multirow{2}{*}{10074} & Costa Rica & $37,2 \%$ \\
\hline & & Otros & $62,2 \%$ \\
\hline \multirow{2}{*}{2013} & \multirow{2}{*}{15918} & Costa Rica & $36,8 \%$ \\
\hline & & Otros & $62 \%$ \\
\hline \multirow{2}{*}{2014} & \multirow{2}{*}{13451} & Costa Rica & $25,9 \%$ \\
\hline & & Otros & $73,8 \%$ \\
\hline \multirow{2}{*}{2015} & \multirow{2}{*}{19931} & Costa Rica & $28,9 \%$ \\
\hline & & Otros & $70,4 \%$ \\
\hline \multirow{2}{*}{2016} & \multirow{2}{*}{15486} & Costa Rica & $24 \%$ \\
\hline & & Otros & $75,4 \%$ \\
\hline \multirow{2}{*}{2017} & \multirow{2}{*}{21692} & Costa Rica & $19,7 \%$ \\
\hline & & Otros & $80,2 \%$ \\
\hline \multirow{2}{*}{2018} & \multirow{2}{*}{39855} & Costa Rica & $13,5 \%$ \\
\hline & & Otros & $86,4 \%$ \\
\hline \multirow{2}{*}{2019} & \multirow{2}{*}{37050} & Costa Rica & $11,7 \%$ \\
\hline & & Otros & $88,1 \%$ \\
\hline
\end{tabular}

Nota: Datos de "Estadísticas de visitas" de Google Analytics, 2019.

Es importante destacar que las visitas en la página web implican las descargas de cada uno de los artículos y también la visualización de estos sin descarga, estadísticas que no se tienen a la mano por el momento. Aunado a esto se puede hablar de las lecturas y descargas que se realizan desde los repositorios ya mencionados, además de la difusión de la versión impresa en los centros de documentación donde se distribuye. 


\section{El aporte de la Revista científica desde la universidad pública}

Es importante considerar que las revistas científicas por sus características se constituyen en canales de información y difusión del conocimiento de gran importancia, en este caso una publicación periódica en el ámbito de los derechos humanos difunde el conocimiento en esta área, además, promueve la investigación y la reflexión crítica, lo cual es influenciado en gran manera por la visibilidad que tiene.

La Revista como una forma de comunicación científica permite dar a conocer el conocimiento que se genera en la universidad y, de esta con la sociedad, en un diálogo de saberes imprescindible de difundir y promover. Así, la comunicación científica está, según Córdoba (2010), en el corazón de la ciencia, de manera tal que los conocimientos deben ser comunicados para generar nuevas formas de saberes, nuevas ideas de investigación y aportes para mejorar la realidad en el área de los derechos humanos desde la universidad.

De esta manera, se puede decir que la revista científica es un espacio que potencia la comunicación para generar un pensamiento crítico, una forma de reivindicar el derecho a la información, al conocimiento, la ciencia y la cultura. De acuerdo con Román (2001, citado por Córdoba, 2010), se puede hablar de tres razones por las cuales la revista científica se cataloga como un vehículo para la ciencia: porque certifica la calidad de los textos que comunica, protege a autores y autoras legalmente (sus derechos de autoría) y archiva la información para facilitar la consulta, procesos que están respaldados por la universidad. En este sentido, la universidad está contribuyendo a una labor esencial de la publicación científica, la cual "asegura el patrimonio científico de la humanidad y favorece la generación y aplicación del conocimiento” (Córdoba, 2010, p. 58).

Asimismo, es un aporte a la sociedad que hace la universidad pública, el cual no solo se constituye como espacio de confluencia de ideas, de conocimientos y propuestas, sino que es un espacio de participación, de libertad de expresión y de pensamiento, se constituye, como un espacio de saberes inter y multidisciplinarios. Con ello, no solo cumple un papel preponderante en la divulgación del quehacer académico, de extensión y de investigación que realiza la universidad editora, sino también incorpora otras universidades latinoamericanas, dando una mayor visibilidad al trabajo de la universidad en el área de los derechos humanos.

Aunado a esto, es importante mencionar que la publicación científica se constituye como un espacio por medio del cual la universidad pública puede acercarse a la sociedad para dar a conocer su trabajo en extensión, investigación y docencia, y 
viceversa, la sociedad puede difundir los saberes, las críticas y las propuestas sobre la realidad.

\section{Conclusión}

El IDELA como instituto que promueve los derechos humanos desde la universidad pública ha impulsado la Revista desde el 2009, de forma que se ha ido convirtiendo, a lo largo de los años, en una publicación de prestigio y calidad, la cual, además, de ser un espacio que permite la difusión del trabajo académico de la universidad en Costa Rica, ha trascendido las fronteras, lo cual la incorpora a las dinámicas de cambio. Este cambio ha motivado a difundir la información para que esta sea más accesible, lo que ha impulsado su cambio al formato de revista electrónica de acceso abierto, el cual contribuye a consolidar el derecho al acceso a la información y la difusión del conocimiento como parte del gran engranaje de la libertad de expresión en América Latina.

Es importante mencionar que el rol que cumplen las universidades públicas en América Latina es de gran importancia para el debate de ideas, la descolonización, el pensamiento crítico, la investigación y las nuevas propuestas, lo cual queda reflejado al ver las contribuciones que se publican en la Revista, mayoritariamente, desde la academia. Asimismo, el aporte desde los diversos países y las diferentes perspectivas enriquece las formas en que se mira la realidad, y no es limitada por una visión meramente endógena del país en la cual se edita.

La Revista enfrenta muchos retos, pues hay muchos caminos que recorrer, vale la pena mencionar que es necesario aunar esfuerzos, ya que, aunque muchas de las personas autoras mencionan que el trabajo es parte de su tesis o de su proceso de formación en una maestría o doctorado, es necesario la presencia de textos cuyas autorías sean estudiantes de pregrado, de igual forma resulta importante la participación de docentes de primaria y de secundaria. Asimismo, es importante destacar la necesidad de ampliar las publicaciones que abarquen campos de estudio en otros países de América Latina y el Caribe, especialmente porque no hay abordajes de países del Caribe y de Centroamérica (con excepción de Costa Rica), de igual manera en el caso de Suramérica se aborda más Argentina y Colombia.

Se debe fortalecer la difusión, para que las visitas en la web se extiendan a otros países, y esto contribuya a tener más colaboraciones, desde otras latitudes, con perspectiva de derechos humanos en América Latina. 


\section{Referencias}

Córdoba, S. (Julio-diciembre, 2010). La comunicación científica. Revista Facultad de Ciencias Médicas, 7(2), 57-61. Recuperado de http://www.bvs.hn/RFCM/pdf/2010/pdf/RFCMVol7-2-2010-8.pdf

DEHUIDELA: Revista de Derechos Humanos, 17. (2008). Heredia: IDELA.

DEHUIDELA: Revista de Derechos Humanos, 18. (2008). Heredia: IDELA.DEHUIDELA. Derechos Humanos IDELA (1999-2007). Heredia: IDELA.

Facultad de Filosofía y Letras (FFL) (2017). Misión, Visión, Valores. Página web Facultad de Filosofía y Letras, UNA. Recuperado de http://www.facultadfilosofia.una.ac.cr/index.php/ informacion-general/valores

Google Analytics. (2019). Estadísticas de visitas. Revista Latinoamericana de Derechos Humanos. Recuperado de https://www.revistas.una.ac.cr/index.php/derechoshumanos/index

IDELA. (2017). Sobre el IDELA. Historia. Página web del IDELA. Recuperado de http://www. idela.una.ac.cr/index.php/sobre-el-idela/historia

IDELA. (2017). Misión del IDELA. Página web del IDELA. Recuperado de http://www.idela. una.ac.cr/index.php/sobre-el-idela/mision

Revista Latinoamericana de Derechos Humanos. (2009-2019). Recuperado de https://www. revistas.una.ac.cr/derechoshumanos 
